\title{
Responsabilidad social universitaria y satisfacción laboral del personal administrativo en una universidad pública de Huancayo (Perú)
}

\author{
Efraín Fernando Miranda Suárez \\ niarfems@hotmail.com \\ David Raúl Hurtado Tiza \\ dahuti.epg7@gmail.com \\ Evita Marisol Fernández Villanueva \\ emarisolfv@hotmail.com \\ Diógenes Orlando Hurtado Tiza \\ hytod@hotmail.com \\ Jouvet Grimanesa Flores Durán \\ yuve_22@Hotmail.com
}

\section{RESUMEN}

El estudio tiene por objetivo de determinar la relación entre responsabilidad social universitaria (RSU) y la satisfacción laboral (SL) del personal administrativo de la Universidad Nacional del Centro del Perú (UNCP), con el fin de mejorar condiciones laborales y de rendimiento. Para ello, se aplicó la metodología cuantitativa, de tipo básica, a un nivel descriptivo y con diseño correlacional. La población de estudio estuvo conformada por los trabajadores administrativos del gobierno central, facultades y sedes de la UNCP, Huancayo - Perú. Así mismo, en la investigación se utilizó la técnica de la encuesta y como instrumento el cuestionario de 18 ítems para los datos de RSU y otro instrumento de 21 ítems para la SL, los cuales fueron validados por exertos y teniendo una confiabilidad de los mismo con Alfa de Cronbach de 0,870 y 0,845 respectivamente. La conclusión a la que se arriba es que existe una correlación débil y significativa entre la responsabilidad social universitaria y la satisfacción laboral del personal administrativo de la Universidad Nacional del Centro del Perú - 2018, con un nivel de significancia de 0,01 y Rho de Spearman de 0.372 .

Palabras clave: RSU; Motivación; Satisfacción; Capacitación; Supervisión. 


\title{
University social responsibility and job satisfaction of administrative staff in a public university in Huancayo (Peru)
}

\begin{abstract}
:
The objective of the study is to determine the relationship between university social responsibility (RSU) and job satisfaction (SL) of the administrative staff of the National University of the Center of Peru (UNCP), in order to improve working conditions and performance. For this, the quantitative methodology, of a basic type, was applied at a descriptive level and with a correlational design. The study population was made up of administrative workers from the central government, faculties and headquarters of the UNCP, Huancayo - Peru. Likewise, in the research the survey technique was used and the 18-item questionnaire was used as an instrument for the RSU data and another instrument of 21 items for the LS, which were validated by experts and having a reliability of the same with Cronbach's alpha of 0.870 and 0.845 respectively. The conclusion reached is that there is a weak and significant correlation between university social responsibility and job satisfaction of the administrative staff of the National University of the Center of Peru - 2018, with a level of significance of 0.01 and Rho of Spearman of 0.372 .
\end{abstract}

Keywords: MSW; Motivation; Satisfaction; Training; Supervision

Artículo recibido: 15. agosto. 2020 Aceptado para publicación: 12. setiembre. 2020 Correspondencia: dahuti.epg7@gmail.com Conflictos de Interés: Ninguna que declarar 


\section{INTRODUCCIÓN}

La universidad como institución es la encargada de: reunir, crear, validar y distribuir la cultura universal. Nacida para el cultivo de las humanidades con el tiempo pasó a ser forjadora del desarrollo disciplinario que la modernidad demandaba. Se inició, formando elites requeridas para la administración de la iglesia, luego con la reforma napoleónica pasó a formar la burocracia que el aparato estatal requería y con el modelo Humboltiano asume la tarea de desarrollar la investigación en las diversas ciencias como función fundamental (Tünnermann, 1998)

En tanto que la universidad se acomodaba a los requerimientos de la demanda de los tiempos, también sus estructuras organizativas fueron ampliándose apareciendo un grupo dedicado únicamente a ver la parte administrativa junto a una plana de docentes.

La administración universitaria se incrementó con la cobertura a la demanda de estudiantes provenientes de las clases medias a sus aulas en la necesidad de profesionalizarse y lograr empleabilidad.

En estas condiciones las funciones fundamentales de la universidad en América latina después de la Reforma de Córdova de 1918 se consolidaron en: la formación profesional, la investigación y la proyección social (Tünnermann, 1998). La comunidad universitaria está conformada por: docentes, autoridades y estudiantes a las que se les reconoce a cada una de ellas como los estamentos universitarios por lo mismo al amparo de la normatividad y la autonomía comparten el cogobierno. En ésta no son considerados los trabajadores administrativos, son vistos como un sector secundario de apoyo al funcionamiento y cumplimiento de la misión de la universidad.

Los estudios de responsabilidad social universitaria (RSU) emprendidos por Vallaeys (2008), Vallaeys, De la cruz y Sasia (2009), Gaete (2011), entre otros, muestran que la universidad genera cuatro impactos como consecuencia de su funcionamiento: 1. impactos organizacionales en las que se incluye la gestión ambiental a la que Gargantini (2014) considera como un quinto impacto o ámbito, 2. Impactos educativos, 3. Impactos cognitivos y 4. Impactos sociales. Como es de observar implícitamente se reconoce cuatro funciones de la universidad; la organizacional más 
allá de las tres últimas reconocidas tradicionalmente. En este sentido "La Responsabilidad Social Universitaria es una política de mejora continua de la Universidad hacia el cumplimiento efectivo de su misión social mediante 4 procesos: Gestión ética y ambiental de la institución; Formación de ciudadanos responsables y solidarios; Producción y Difusión de conocimientos socialmente pertinentes; Participación social en promoción de un Desarrollo más humano y sostenible" (Vallaeys, 2008, p. 210).

Lo apuntado demuestra que la satisfacción laboral expresado en sus dimensiones: clima institucional, condiciones de trabajo y movilidad social, es una resultante entre lo emocional y lo afectivo surgido de una identidad positiva como reacción comportamental del trabajador universitario con su centro laboral la universidad donde laboral, y a partir de ella surge un compromiso que lo hace copartícipe de los objetivos y problemas institucionales.

De lo anterior surgió la necesidad de investigar ¿cuál y cómo es la relación existente entre responsabilidad social universitaria y satisfacción laboral del personal administrativo de la Universidad nacional del Centro del Perú. Huancayo 2018?. El objetivo general de la investigación es identificar la relación existente entre la responsabilidad social universitaria y la satisfacción laboral del personal administrativo de la Universidad Nacional del Centro del Perú, Huancayo 2018. Y como hipótesis general fue: Existe relación significativa entre la responsabilidad social universitaria y la satisfacción laboral del personal administrativo de la Universidad Nacional del Centro del Perú, Huancayo 2018. Para, los problemas, objetivos e hipótesis especificas correlacionales, se realizó de variable a dimensión. Es decir, la variable Responsabilidad Social Universitaria (RSU) con cada dimensión de Satisfacción Laboral (SL).

\section{ESTRATEGIAS METODOLÓGICAS O MATERIALES Y MÉTODOS}

La investigación responde a la metodología cuantitativa, para ello se siguieron los pasos del método científico, descriptivo, estadístico, hipotético y deductivo. La 
tipología de estudio obedece al básico, ya que la finalidad era de conocer y contrastar las teorías de la Responsabilidad Social Universitaria (RSU) y Satisfacción Laboral (SL) en un contexto dado, el nivel y diseño de investigación fue descriptivo y correlacional respectivamente, donde se buscó la correlación o asociación entre ambas variables (Hernández, Fernández y Baptista, 2014) y Sierra (2008).

\section{Participantes}

La investigación se realizó en la Universidad Nacional del Centro del Perú de la provincia de Huancayo, Perú. La población accesible de estudio estuvo conformada por los administrativos pertenecientes de las oficinas de gobierno central (17), sede 1 (6), sede 2 (7) y Facultades (22), haciendo un total de 52 administrativos. Para ello, se consideró el criterio de inclusión como la participación voluntaria y encuestas rellenadas correctamente; excluyéndose a los que contradicen lo mencionado. Asimismo, él estudió protegió la identificación de los encuestados, las cuales se encuentran en completa reserva sus nombres y cargos que desempeñan en universidad. La población accesible de estudio fue teniendo en cuenta los criterios de inclusión y exclusión, que dando conformada como una muestra censal de 52 administrativos de las diferentes áreas de la UNCP, 2019 (gobierno central y facultades).

\section{Instrumentos}

Para medir ambas variables se utilizaron la técnica de encuesta y como instrumento el cuestionario, los cuales fueron validadas mediante juicio de experto, opinando que dichos instrumentos cumplen con la coherencia entre la variable y dimensión, dimensión e indicadores; indicadores e ítem; ítem y opción de respuesta. Asimismo, la confiabilidad de consistencia interna, según el estadígrafo Alfa de Cronbach del cuestionario de responsabilidad social universitaria es de 0,870 y del cuestionario de satisfacción laboral es de 0,845 .

El cuestionario de Responsabilidad Social Universitaria fue extraído del primer componente del manual de primeros pasos propuesto por Vallaeys (2009), tiene 18 ítems las cuales están distribuidas en las dimensiones: clima laboral (6 ítems); campo ecológico (6 ítems); y ética y transparencia (6 ítems). 
El cuestionario de satisfacción laboral fue adaptado por Moisés Fernando Flores Gavino en el 2015, validado mediante juicio de expertos y la fiabilidad mediante Rolun y Gutman obtuvo 0,870. El cuestionario, tiene 21 preguntas las cuales están distribuidas en las dimensiones: motivación (5 ítems); satisfacción personal (6 ítems); capacitación del servidor (5 ítems) y supervisión (5 ítems).

La escala utilizada fue el tipo Likert para ambas variables, la confiablidad se realizó mediante una prueba piloto de 15 administrativos y con el método de consistencia interna, el Alfa de Cronbach obtenido para RSU fue de 0,970 y de SL de 0,871. Finalmente, para medir el nivel de las dimensiones y variables en ambas se realizó la baremación, teniendo en cuenta el puntaje máximo, mínimo y opción de respuesta. (Triolla, 2008).

\section{Procedimientos}

Para la aplicación de los instrumentos se procedió con el protocolo de investigación, solicitando la autorización respectiva en la instancia correspondiente. El instrumento pasó antes por el proceso de validez (juicio de experto) y confiabilidad (prueba piloto/consistencia interna), con ello, se aplicó los instrumentos a los encuestados, donde se dio a conocer el objetivo de la investigación e instrumento, como también las instrucciones para su rellenado. Una vez terminada el recojo de información, se pasó a elaborar la base de datos, procesar y analizar en el programa estadístico SPSSV25 y Microsoft Excel.

\section{RESULTADOS Y DISCUSIÓN}

Los métodos de análisis de datos fueron teniendo encueta le objetivo, hipótesis, tipo de investigación y la escala de medición. Los resultados descriptivos se presentaron con tablas y figuras. Para determinar el nivel de cada una de las variables se baremo y para identificar la correlación se utilizó Rho de Spearman y para la prueba de significancia para la validez de las hipótesis y significancia Rho de Spearman (Correlación entre ambas variables) 


\section{RESULTADOS}

Los resultados generales encontrados de la aplicación de las encuestas validadas y

fiables de la variable "Responsabilidad Social Universitaria” y “Satisfacción laboral” en los administrativos de la Universidad Nacional del Centro del Perú. A continuación, se muestra en la siguiente:

\section{Tabla 01}

Nivel de responsabilidad social universitaria

\begin{tabular}{lcc}
\multicolumn{1}{c}{ Nivel } & Frecuencia & Porcentaje \\
\hline Bajo: $18-36$ & 0 & 0.00 \\
Medio: 36 - 54 & 28 & 53.85 \\
Bueno: 54 - 72 & 24 & 46.15 \\
Muy Bueno: 72 - 90 & 0 & 0.00 \\
Total & 52 & 100.00 \\
\hline
\end{tabular}

Fuente: Bases de datos de los investigadores.

En la tabla 1, se aprecia que el 53,85\% de administrativo opinan que la responsabilidad social universitaria se encuentra en el nivel medio, por percibir un clima laboral en proceso en cuanto medición y mejora continua, participación del personal en las diferentes metas, círculos de dialogo y el respeto a los derechos laborales del trabajofamilia de está. Asimismo, manifiestan que el nivel es medio en referencia a la dimensión campos ecológico, porque consideran que la universidad está recién implementando un sistema de gestión ambiental integral y política en el cuidado ambiental en sus contrataciones con terceros de manera responsable.

Finalmente, opinaron en cuanto a la ética y transparencia se encuentra en este nivel, porque pese a ver buenas iniciativas sobre el aspecto ético esto se debe normar en todas las instancias y que la actuación de todos los funcionarios, administrativos debe ser transparente, denunciando a las instancias correspondientes sobre cualquier indicio o acto de corrupción, donde se respetan y se fomenta la participación democrática de toda la comunidad universitaria. 
Tabla 2

Nivel de satisfacción laboral

\begin{tabular}{lcc}
\hline Nivel & Frecuencia & Porcentaje \\
\hline Bajo: $21-42$ & 0 & 0.00 \\
Medio: $42-63$ & 41 & 78.85 \\
Bueno: $63-84$ & 8 & 15.38 \\
Muy Bueno: $84-105$ & 3 & 5.77 \\
Total & 52 & 100.00 \\
\hline
\end{tabular}

Fuente: Base de datos de la satisfacción laboral

Como se aprecia en la tabla 2, los resultados del nivel de satisfacción laboral son medio con 78,85\% según los administrativos de la Universidad Nacional del Centro del Perú. Señalan que dicho nivel es porque aún están en proceso de mejora el sistema de información que se practica en las diferentes áreas administrativas. Asimismo, tener una política de estímulos, reconocimiento y promoción para el desarrollo laboral, económico, socio afectivo e institucional.

Ahora se ha calculado la correlación entre ambas variables, mediante la prueba de hipótesis de significancia, con el estadígrafo de Spearman es:

Tabla 3

Correlación de responsabilidad social universitaria y satisfacción laboral

\begin{tabular}{|cll|r|r|}
\hline & & $\begin{array}{l}\text { Responsabilidad } \\
\text { Social Universitaria }\end{array}$ & $\begin{array}{c}\text { Satisfacción } \\
\text { Laboral }\end{array}$ \\
\hline $\begin{array}{c}\text { Spearman's } \\
\text { rho }\end{array}$ & $\begin{array}{l}\text { Responsabilidad } \\
\text { Social }\end{array}$ & Correlation & 1.000 & $.372(* *)$ \\
& Universitaria & Coefficient & & \\
& & Sig. (2-tailed) &. & .000 \\
& & $\mathrm{~N}$ & 52 & 52 \\
& Satisfacción & Correlation & $.372(* *)$ & 1.000 \\
& laboral & Coefficient & & \\
& & Sig. (2-tailed) & .000 & .52 \\
\hline
\end{tabular}

** Correlation is significant at the 0.01 level (2-tailed).

Como se aprecia en la Tabla 3, la correlación estadísticamente entre ambas variables según la rho de Spearman es de 0,372 a un nivel de significancia de 0,01. Esto, en 
concordancia a lo que indican, Hernández, Fernández y Baptista, (2010) sobre la equivalencia de dicho valor se encuentra como una correlación entre ambas variables es positiva débil. Para la docimasia de la hipótesis general se siguieron los siguientes pasos: Planteamiento de hipótesis estadístico: $\mathrm{H}_{\mathrm{o}}$ : No existe una relación significativa entre ambas variables. $\mathrm{H}_{1}$ : Existe una relación significativa entre ambas variables. El nivel de significancia o riesgo considerado es de 0,05; Según los resultados visto en la tabla indican que hay una correlación positiva débil entre ambas variables y que el nivel de significancia es menor a lo planteado. Por tanto, se rechazó la hipótesis nula y se acepta la hipótesis alterna. Este resultado muestra que existe una relación débil y significativa entre la responsabilidad social universitaria y la satisfacción laboral en los administrativos en la UNCP.

Con ello se contrasto con otros los trabajos como de: Montoya, Bello, Bermúdez, Burgos., Fuentealba, M. y Padilla, (2017). Asimismo, Pecino, Mañas, Díaz, López, y Llopis, (2015). Regalado, (2016); Rojas y Távara (2017). Ticse, (2019), quienes e aprecia que estudiaron la relación de satisfacción laboral y RSU, sustentada en la determinación del clima laboral que la universidad propicia para sus trabajadores. Estos están dispuestos a mayor identidad, laboriosidad y proactividad porque la universidad les proporciona posibilidades de realización de movilidad laboral, aun cuando se ha comprobado que la laboral mide RSU en estas universidades aún están en términos iniciales, por su adecuación a la nueva ley 30220.

De la misma manera, los investigadores como: Montoya, Bello, Bermúdez, Burgos., Fuentealba, M. y Padilla, (2017). Asimismo, Pecino, Mañas, Díaz, López, y Llopis, (2015). Regalado, (2016); Rojas y Távara (2017). Ticse, (2019). Asimismo, Ramírez y Paz, (2010), quienes estudiaron la relación de satisfacción laboral y RSU, se sustenta en la determinación del clima laboral que la universidad propicia para sus trabajadores. Estos están dispuestos a mayor identidad, laboriosidad y proactividad porque la universidad les proporciona posibilidades de realización de movilidad laboral, aun cuando se ha comprobado que la laboral mide RSU en estas universidades aún están en términos iniciales, por su adecuación a la nueva ley 30220. 
Al respecto, Davis y Newstrom (2003), definen la satisfacción laboral como el conjunto de sentimientos y emociones favorables o desfavorables que los trabajadores ven a su trabajo. De tal modo que está es relacionada con tres actitudes distinguibles en los empleados: 1. La dedicación al trabajo, que tiene que ver directamente el insumo del tiempo y energía no simplemente en el cumplimiento de sus tareas sino la dedicación, el empeño, la calidad y ética de sus servicios; 2. El compromiso organizacional, entendido como la identidad con la institución donde labora que la considera como suya y se esfuerza por el logro de los objetivos, visión y misión institucional, se conduce por las políticas de su centro laboral y está dispuesto a permanecer por largas temporadas; y finalmente 3. El estado de ánimo en el trabajo, implica: entusiasmo, energía, proactividad y trabajo en equipos, saliendo de la rutina y la automatización.

Los otros resultados relacionados a los objetivos e hipótesis específicas indican que existe relación significativa débil entre la responsabilidad social universitaria con la motivación (rho=0,312); satisfacción personal $(\mathrm{rho}=0,261)$; capacitación del servidor (rho=0,303) y la supervisión (rho=0,271) según los administrativos del gobierno central, facultades y sedes de la UNCP, respectivamente. Ello, nos hacen reflexionar que hay tarea pendiente de nuestras autoridades universitarias en sensibilizar, empoderar e implementar una política de gestión bajo la mirada de la RSU en los actores administrativos que son parte del grupo de interés interno.

La universidad como institución que ha asumido el rol histórico de preservar, ampliar, validar y distribuir el conocimiento universal, es una entidad compleja. Atrás quedaron los tiempos en que su función era la relación directa entre el docente (catedrático) y el discente (discípulo, estudiante o alumno). Con la modernidad la universidad paso a masificarse y con ella surgió la necesidad de requerir grupos profesionales especializados en la administración en sus distintas especializaciones. Estos grupos son los que en la actualidad constituyen el sector de los llamados trabajadores universitarios no docentes o administrativos universitarios (Davis y Newstrom, 2003). 
La labor que cumplen estos trabajadores universitarios se ha ido especializando a la velocidad del crecimiento y modelo que adopta cada institución. Sin embargo, esta plana burocrática es la que se encarga de la administración y es responsable del funcionamiento y cumplimiento de los objetivos estratégicos institucionales, llevando control de todo el movimiento de la economía, bienes muebles e inmuebles y el personal docente y no docente que trabaja en la universidad, así como del alumnado (Luna, 2015).

Al ser incorporados a la universidad los trabajadores empiezan a establecer nuevos procesos de socialización en cuyo discurrir surgen niveles de integración como de exclusión voluntaria o involuntaria. Nace en ellos identidades y descontentos, expectativas como frustraciones personales, todas ellas como resultado del clima institucional, la relación entre ellos y las autoridades, las jerarquías del sistema organizacional, de las labores que cumplen, así como las expectativas de orden económico o movilidad laboral entre otros. De modo que para negociar y solucionar algunos conflictos y alcanzar beneficios diversos como sujetos de derecho amparados por la legislación laboral vigente llegan a conformar gremios.

En una universidad socialmente responsable los trabajadores son considerados como el intangible en el respeto de los derechos humanos y las normas laborales de la OIT y del país. Busca un óptimo clima laboral para el mejor rendimiento del capital humano y gestiona los recursos humanos con procesos democráticos.

De otro lado, De la Cruz y Sasia, (2008). Mencionan que no solo el trabajador universitario es considerado como individuo aislado, sino como integrante de una familia, por lo mismo demanda y adquiere derechos para protegerlos, en esa dimensión de responsabilidad social la universidad promoverá políticas de inclusión familiar. De las condiciones materiales que la universidad promueve para el trabajador y su familia dependerá la satisfacción y la identidad y compromiso con su trabajo, lo que generará mayor producción y productividad en la función encomendada lo que contribuirá a alcanzar los objetivos estratégicos. En la cotidianidad los trabajadores con los procesos de interacción, socialización empiezan a internalizar las normas visión y misión de la 
universidad van construyendo una cultura organizacional, así resulta como señalan (Ramírez y Paz, 2010)

Resulta de interés una cultura de identidad con el desempeño a través de sus responsabilidades y funciones en el cargo ocupado, mostrando un sentido de pertenencia aunado a la satisfacción laboral con la organización; mediante el reconocimiento de sus necesidades como trabajador, producto de los aspectos derivados del propio trabajo o el grado en el cual se consiguen determinados resultados o beneficios, lo cual puede afectar tanto la salud física, como el bienestar psicológico personal o colectivo.

\section{CONSIDERACIONES FINALES}

1. Se identificó que existe relación débil y significativa entre la responsabilidad social universitaria y la satisfacción laboral del personal administrativo de la Universidad Nacional del Centro del Perú - 2018, a un nivel de significancia de 0,01 y rho =0,372.

2. Se identifico que existe relación débil y significativa entre la responsabilidad social universitária y la motivación del personal administrativos de la Universidad Nacional del Centro del Perú - 2018, a un nivel de significancia de 0,01 y rho =0,312

3. Se identificó que existe relación débil y significativa entre la responsabilidad social universitaria y la satisfacción personal de los administrativos de la Universidad Nacional del Centro del Perú, con un nivel de significancia de 0,01 y rho =0,261.

4. Se identificó que existe relación débil y significativa entre la responsabilidad social universitaria y la capacitación del servidor del personal administrativo de la Universidad Nacional del Centro del Perú, con un nivel de significancia de 0,01 y rho $=0,303$.

5. Se identificó que existe relación débil y significativa entre la responsabilidad social universitaria y la supervisión del personal administrativos de la Universidad Nacional del Centro del Perú, con un nivel de significancia de 0,01 y rho =0,271. 


\section{LISTA DE REFERENCIAS}

Argandoña, A. (2007), Responsabilidad social universitaria de la Empresa. Fundación Caritas España. Española editores.

Baltera, P. y Díaz, E. (2005), Responsabilidad social universitaria Empresarial. México Ediciones dirección del trabajo.

Davis, K. y Newstrom, J. (2003), Comportamiento Humano en el Trabajo. México. Editorial Mcgraw-hill Interamericana. México.

De la Cruz y Sasia, (2008). La responsabilidad de la universidad en el proyecto de construcción de una sociedad, en: Revista Educación Superior y Sociedad, 13(2) $: 17-52$.

Gaete, R. (2011). La responsabilidad social universitaria como desafío para la gestión estratégica de la Educación Superior: el caso de España, en: Revista de Educación, N. 355 :109-133.

Gargantini, D. (2014) Estado de la responsabilidad social universitaria: El aporte de AUSJAL a la institucionalización del enfoque en América latina. Cuadernos de RSO. Universidad Católica del Uruguay. Montevideo.

Guedez, V. (2006), Ética y Práctica de la Responsabilidad social universitaria Empresarial. Venezuela. Editorial Planeta.

Hernández, R.; Fernández, C. y Baptista, P. (2010) Metodología de la Investigación. México. Editorial Mc. Graw Hill Interamericana Editores, S.A. Quinta Edición.

Hernández, R.; Fernández, C. y Baptista, P. (2014) Metodología de la Investigación. México. Editorial Mc. Graw Hill Interamericana Editores, S.A. Sexta Edición

Licha, I. (2012). La responsabilidad social de la educación superior, pp.519-537. En: Licha, I. (Comp.) Enseñanza de la responsabilidad social empresarial Retos de las universidades en Iberoamérica. Buenos Aires: PNUD.

Luna, M. (2015). Satisfacción laboral y compromiso organizacional del personal docente de una Universidad Privada de Lima. Tesis de Grado - UIGV. http://repositorio.uigv.edu.pe/handle/20.500.11818/385

Nuñez, M; Salom, J; Paz, A; Rosales, V. (2012) Responsabilidad social universitaria Universitaria: Enfoque de Gestión Ética Compartida. Venezuela Revista Opción. Volumen 28. 2012. No. 69 
Ramírez, L. y Paz, A. (2010) Responsabilidad social y satisfacción laboral en universidades privadas. Ponencia en VII Jornadas Nacionales y IV Internacionales de Investigación de la URBE, Venezuela.

Robbins, S. (2004), Comportamiento Organizacional. México. Editorial Prentice Hall Interamericana.

Montoya, P., Bello, N.; Burgos, F.; Fuente, A.; Padilla, A. (2017). Satisfacción Laboral y su relación con el clima organizacional en funcionarios de una universidad estatal chilena. Articulo para Ciencia y Trabajo 2017. N58.Chile

Vallaeys, F.; De la Cruz, C y Sasia, P. (2009). Responsabilidad social universitaria universitaria: manual de primeros pasos. México. Editorial McGraw-Hill Interamericana Editores, s.a. de C.V.

Vallaeys, F. (2003). Marco Teórico de Responsabilidad social universitaria Universitaria. Editorial texto C.A. Caracas

Pecino, V.; Mañas, M., Díaz, P., López, J. y Llopis, J. (2015). Clima y satisfacción laboral en el contexto universitario. Anales de psicología, 2015, vol. 31, N. 2 (mayo). Edición impresa: 0212-9728. ISSN Universidad de Almería 2 Universidad Católica San Antonio de Murcia. España.

Ticse, F. (2019) Evaluación del desempeño y satisfacción laboral en los servidores públicos no docentes de la Universidad Nacional Daniel Alcides Carrión 2017.Cerro de Pasco - Perú

Tunnermann, C. (2003) La Universidad latinoamericana ante los retos del siglo XXI.

Colección UDUAL. Gisela Rodríguez. México. 